\section{Genomic segmental polymorphisms in inbred mouse strains}

Jiangzhen $\mathrm{Li}^{1,6}$, Tao Jiang ${ }^{1,6}$, Jian-Hua Mao ${ }^{2}$, Allan Balmain ${ }^{2}$, Leif Peterson ${ }^{1,3}$, Charles Harris ${ }^{4}$, Pulivarthi H Rao ${ }^{4}$, Paul Havlak ${ }^{1,5}$, Richard Gibbs ${ }^{1,5} \&$ Wei-Wen Cai ${ }^{1}$

By analyzing genomic copy-number differences using highresolution mouse whole-genome BAC arrays, we uncover substantial differences in regional DNA content between inbred strains of mice. The identification of these apparently common segmental polymorphisms suggests that these differences can contribute to genetic variability and pathologic susceptibility.
To facilitate high-resolution and high-throughput analysis of genomic abnormalities in mouse tumors and models of induced chromosomal abnormalities, we developed BAC arrays ${ }^{1}$ to cover the entire mouse genome. The new whole-genome BAC arrays $(19 \mathrm{k}$ arrays) consist of more than 19,200 BAC clones that form a virtually complete tiling path, except in regions without clone coverage in the present mouse physical map ${ }^{2}$. We applied the $19 \mathrm{k}$ BAC arrays to detect chromosomal imbalances in mouse tumor samples from a $129 / \mathrm{Sv}$ background, using normal DNA from C57BL/6J mice as the control, and identified several regions that consistently showed copy-number losses or gains (Fig. 1 and Supplementary Note online).

To investigate the prevalence of these segmental polymorphisms, we analyzed another 14 commonly used inbred mouse strains. We found 216 BACs that consistently showed losses and 130 BACs that consistently showed gains (Supplementary Table 1 online). Notably, some of these regions span more than $1 \mathrm{Mb}$ and were detected by
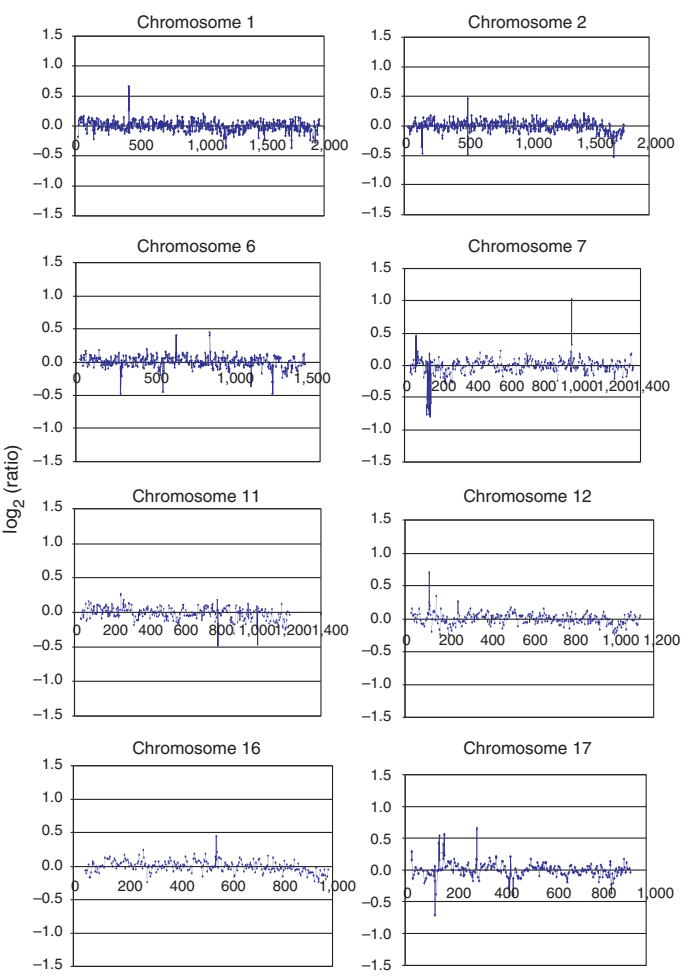

Chromosome 7
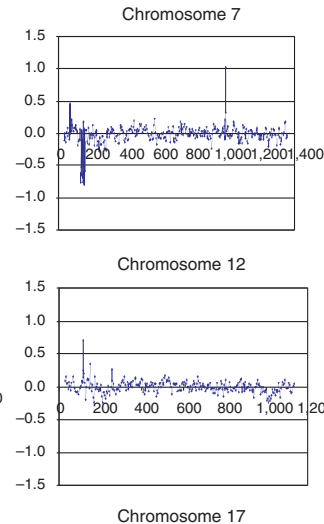

Chromosome 17

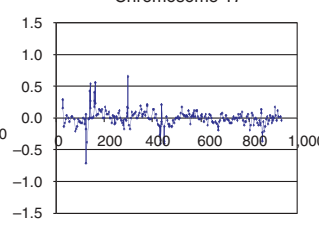

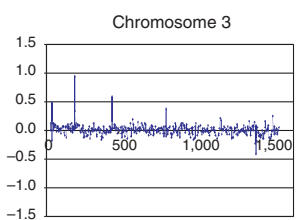

Chromosome 8

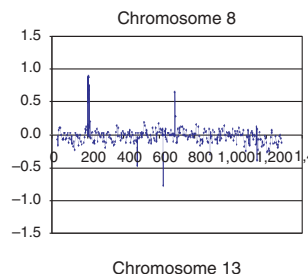

Chromosome 13

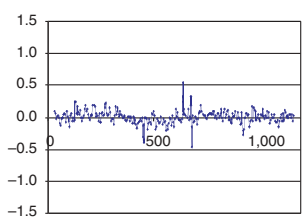

Chromosome 18

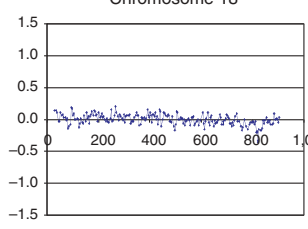

Unit (100 kb)

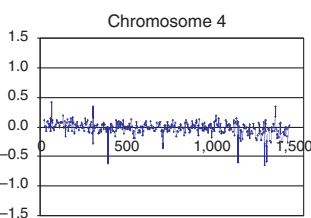

Chromosome 9

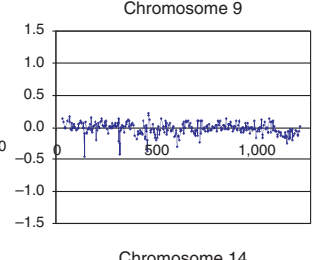

Chromosome 14

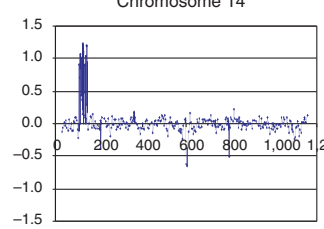

Chromosome 19

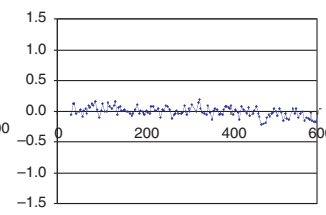

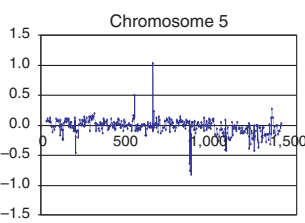

Chromosome 10

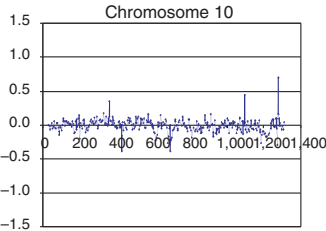

Chromosome 15

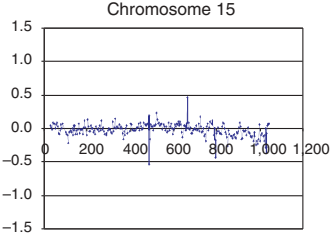

Chromosome X

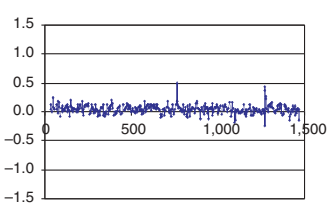

Figure 1 Chromosomal copy-number profiles of inbred mouse strain 129/Sv relative to strain C57BL/6J. The $x$ axis shows chromosome position from centromere to telomere in $100-\mathrm{kb}$ units. The $y$ axis shows the normalized $\log _{2}$ ratio of fluorescence signals in strain $129 / \mathrm{Sv}$ relative to control strain C57BL/6J.

${ }^{1}$ Department of Molecular and Human Genetics, Baylor College of Medicine, One Baylor Plaza, Houston, Texas 77030, USA. ${ }^{2}$ Cancer Research Institute, University of California at San Francisco, 2340 Sutter Street, San Francisco, California 94143, USA. ${ }^{3}$ Department of Medicine, ${ }^{4}$ Department of Pediatrics and ${ }^{5}$ Human Genome Sequencing Center, Baylor College of Medicine, One Baylor Plaza, Houston, Texas 77030, USA. ${ }^{6}$ These authors contributed equally to this work. Correspondence should be addressed to W.W.C. (wcai@bcm.tmc.edu).

Published online 22 August 2004; doi:10.1038/ng1417 
multiple overlapping BAC clones in more than one strain. For example, a region showing gain on chromosome 14 in strain $129 / \mathrm{Sv}$ spanned $\sim 4 \mathrm{Mb}$ and appeared normal in all other strains, except SPRET/EiJ and SENCARA/PtJ, which showed copy-number loss in that region (Fig. 2a). A small region around $36.4 \mathrm{Mb}$ on chromosome 14 showed both loss and gain in multiple strains, whereas a 3-Mb region on chromosome 7 showed loss in most strains tested (Fig. 2b). At 20.1-20.6 Mb on chromosome 8, multiple overlapping BACs indicated copy-number gains in 13 inbred strains, and strain a
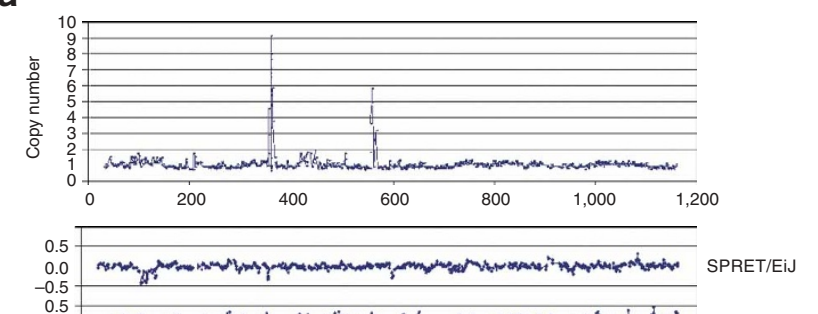

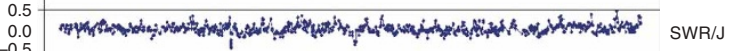

SM/J

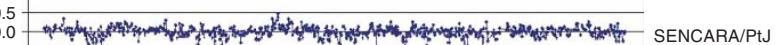

0.5

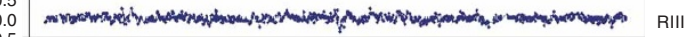

0.5

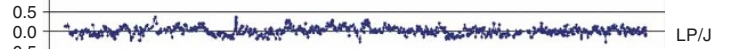

-0.5
0.5

\begin{tabular}{r|r}
0.5 \\
-0.5
\end{tabular}

. \begin{tabular}{rr}
$\frac{0}{ \pm}$ & 0.5 \\
\hline & 0.0
\end{tabular}

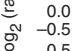

-0.5
0.5
0.0
-0.5
0.5

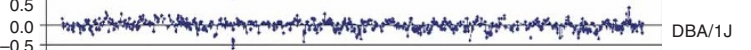

0.5

$\begin{array}{lll}0.5 & 0 \\ -0.5 & & \end{array}$

0.5

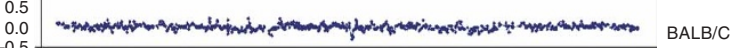

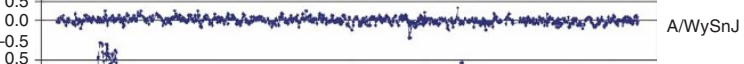

0.0
-0.5

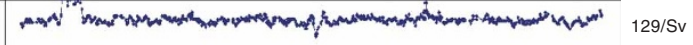

0

200

400

600

800

$1,000 \quad 1,200$

In all BAC array hybridizations, we used genomic DNA from strain $\mathrm{C} 57 \mathrm{BL} / 6 \mathrm{~J}$ as the control and measured the copy-number variation as a significant deviation $(P<0.0012)$ of fluorescence ratios from the baseline level along individual chromosomes. Because all BAC clones of the $19 \mathrm{k}$ arrays carry insert DNA only from the C57BL/6J strain, it is not possible to detect deletions in this strain. Therefore, detection of gains in other strains reflects real DNA copynumber gain. Detection of copy-number losses, however, can be caused by a deletion in the test samples, by a gain in the control sample or by a combination of both.

\section{b}
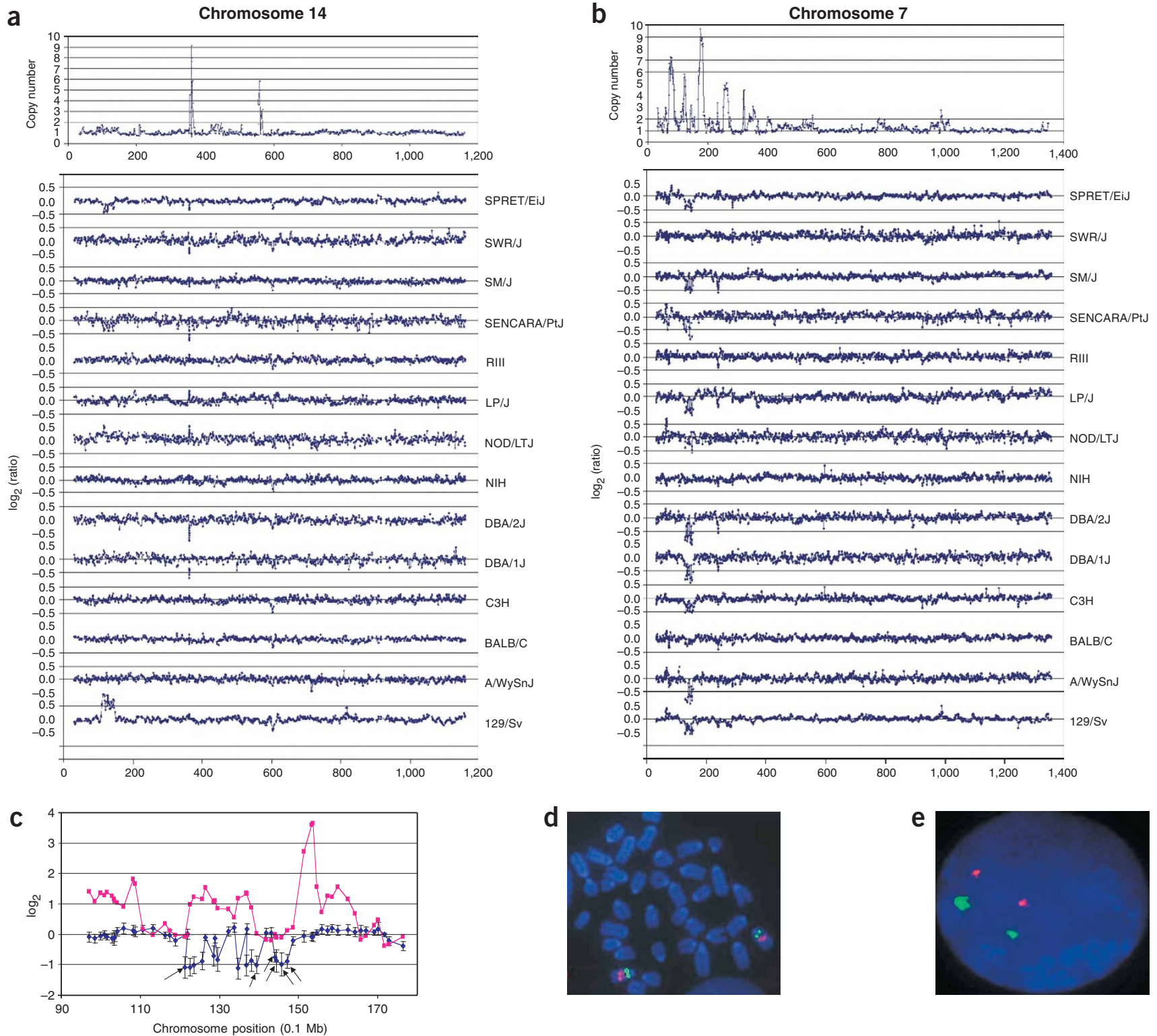

Figure 2 Copy-number profiles of chromosomes 14 and 7 and FISH validation. (a,b) Relative copy-number profiles of chromosomes 14 and 7 in 14 inbred strains (lower panels) compared with the calculated copy-number profile (upper panels) of the same chromosomes in the control strain C57BL6J.

(c) Comparison of sequence copy number of strain C57BL/6J and BAC array-detected relative copy number of strain 129/Sv in the region 9.7-17.6 Mb on chromosome 7. Pink squares, average sequence copy number $\left(\log _{2}\right)$ of individual BAC clones. Blue diamonds, relative copy number ( $\left.\log _{2}\right)$ of strain $129 / \mathrm{Sv}$ versus strain C57BL/6J detected using array comparative genomic hybridization. Data shown are the mean \pm s.d. of 24-28 independent measurements. $(\mathbf{d}, \mathbf{e})$ Metaphase (d) and interphase (e) FISH images of BAC probe RP23-280K1. This clone was one of the six BACs that detected a loss of copy number but were located in regions (indicated by arrows in c) with an apparent single copy in strain C57BL/6J. A mouse hybrid embryonic stem cell line derived from a hybrid of strains 129/Sv and C57BL/6J was used for FISH study. The test probe and control probe (RP23-143L18) were visualized in green and red, respectively. 
To determine whether some of the apparent deletion regions may be due to sequence duplications in the control sample from strain C67BL/6J rather than bona fide deletion in the test samples, we use an oligomer counting approach ${ }^{3}$ (Supplementary Methods online) to analyze the copy-number variations across the genome of the $\mathrm{C} 57 \mathrm{BL} / 6 \mathrm{~J}$ strain. We found that BAC clones showing loss were more frequently $\left(P<0.0023, \chi^{2}\right.$ test $)$ located in regions with higher copy number in strain C57BL/6J, suggesting that the reduced copy number in the test strains may not be caused by real deletion. However, 90\% of BAC clones showing loss were found in regions with apparently normal copy number. These BACs probably contain deletions of segmental sequences that are smaller than the insert of an average BAC clone $(\sim 175 \mathrm{~kb})$. Deletion of large contiguous regions $(>250$ $\mathrm{kb}$ ) should be rare, because we found no BAC clones with a $\log _{2}$ ratio of more than 2.5 and less than -2.5 in dye-reversal experiments, as expected for inbred mouse strains.

To investigate the possible mechanisms underlying such segmental polymorphisms, we further analyzed the sequence features and the flanking regions (within $200 \mathrm{~kb}$ ) of all the BACs that showed copynumber variations. We found that $10 \%$ of BACs showing loss and $1 \%$ of BACs showing gain were associated with segmental duplications ${ }^{4,5}$ (Supplementary Table 2 online); such association was found in only $3 \%$ of randomly selected BACs. The frequent association $(P<0.0005)$ of segmental duplication with BACs showing loss suggests that a recombination-mediated sequence-deletion mechanism, similar to that found in some human genomic disorders ${ }^{6}$, could lead to some 을 of these copy-number variations.

To investigate whether some of the regions showing copy-number variations were complete deletions that were not clearly detected because of BAC cross-hybridizations, we analyzed the clone representation of regions showing loss in strain 129/Sv. A 'gene targeting' library was constructed for this strain and characterized by end sequencing. These clones were randomly distributed through the whole genome, with an average spacing of $39 \mathrm{~kb}$ (ref. 7). We analyzed 31 regions showing loss in strain 129/Sv that are covered by BACs. (2) None showed absence of construct clone coverage, indicating that loss in these regions detected by the BAC arrays is not due to complete loss of large contiguous segments.

Fluorescence in situ hybridization (FISH) experiments also indicated that the detected segmental polymorphisms are probably due to small-scale variations of sequence copy number rather than large contiguous deletions. We selected six BAC clones (Fig. 2c) and two control clones for FISH validation from a region on chromosome 7 (Fig. 2c) that showed frequent loss in multiple strains (Fig. 2b). We hybridized BAC probes to C57BL/6J and 129/Sv hybrid embryonic stem cells. For each of the six probes, we scored 25 metaphase and 50 interphase cells and found similar patterns of hybridization signals. We quantified FISH signals from 7-12 interphase cells and tested the statistical significance of differences in signal intensity between the test probes and the control probe. In each case, the signals from one homolog were significantly $(P<0.0005$, paired $t$-test $)$ stronger than those from the other (Fig. 2d,e). These results fully corroborated the BAC array findings (Fig. 2c).

To confirm the segmental polymorphisms detected by BAC arrays, we used quantitative PCR assays (Taqman) to measure copy number in three regions across all 14 mouse strains. We found a $100 \%$ concordance between results of array comparative genomic hybridization and Taqman assays, using a copy ratio cutoff value of 0.75 with respect to strain C57BL/6J in both methods (Supplementary Fig. 1 and Supplementary Table 1 online).

Common segmental polymorphisms shared between strains are indicators of their evolutionary history. We attempted to analyze the relatedness between the tested strains according to their segmental polymorphisms profiles using unsupervised hierarchical cluster analyses $^{8}$. Even with our relatively limited and low-resolution data on copy-number variation, we were able to stratify the relatedness of mouse strains. Our results were comparable to those derived from high-resolution single-nucleotide polymorphism data ${ }^{9,10}$ (Supplementary Fig. 2 online).

Segmental polymorphisms have been found in humans ${ }^{11,12}$ but none has been reported in inbred mouse strains. Our results indicate that large genomic segmental polymorphisms can be rapidly mapped using high-resolution BAC array comparative genomic hybridization. Application of this array-based high-resolution and high-throughput screening approach, in combination with conventional genetic approaches, will generate more data that will help to establish the biological relevance of genomic segmental polymorphisms.

Note: Supplementary information is available on the Nature Genetics website.

\section{ACKNOWLEDGMENTS}

We thank A. Bradley for the mouse hybrid ER3.4 embryonic stem cell line and Q. Li for technical support in BAC array preparation. This work was support in part by the US Department of Energy.

\section{COMPETING INTERESTS STATEMENT}

The authors declare that they have no competing financial interests.

Received 29 April; accepted 3 August 2004

Published online at http://www.nature.com/naturegenetics/

1. Cai, W.W. et al. Nat. Biotechnol. 20, 393-396 (2002).

2. Gregory, S.G. et al. Nature 418, 743-750 (2002).

3. Havlak, P. et al. Genome Res. 14, 721-732 (2004).

4. Cheung, J. et al. Genome Biol. 4, R47 (2003).

5. Bailey, J.A. et al. Genome Res. 14, 789-801 (2004).

6. Lupski, J.R. Trends Genet. 14, 417-422 (1998).

7. Adams, D.J. et al. Nat. Genet. 36, 867-871 (2004).

8. Peterson, L.E. Comput. Methods Programs Biomed. 69, 179-188 (2002).

9. Wade, C.M. et al. Nature 420, 574-578 (2002).

10. Wiltshire, T. et al. Proc. Natl. Acad. Sci. USA 100, 3380-3385 (2003)

11. Bailey, J.A. et al. Science 297, 1003-1007 (2002).

12. Der-Sarkissian, H. et al. Genome Res. 12, 1673-1678 (2002). 